\title{
Medical genetics: advances in brief
}

C. elegans cell survival gene ced-9 encodes a functional homologue of the mammalian proto-oncogene bcl-2

Hengartner MO, Horvitz RH. Cell 1994;76: 665-76.

Apoptosis or programmed cell death is important both in development and in tissue homeostasis. Recently, the bcl- 2 proto-oncogene has been identified as one of the key genes involved in the regulation of this process and the expression of the gene is associated with either inhibition or delay of apoptosis in various tissues. This paper reports the cloning and characterisation of the nematode Caenorhabditis elegans ced-9 gene which functions in a similar way to the mammalian bcl-2 gene in the negative regulation of cell death process. A polycistronic locus was identified in the ced-9 region which included, in addition to ced-9, a gene (cyt-1) that encodes a protein similar to a mitochondrial cytochrome. Ced-9 protein shows both sequence and structural homology to bcl-2 and bcl-2 like proteins. In addition, bcl-2 proved to be an effective substitute for ced-9 in preventing cell death in both normal and ced-9 deficient mutant nematodes. These findings clearly indicate that bcl- 2 and ced-9 are members of the same family and indicate that programmed cell death as a process developed early in evolution. It is also interesting to speculate on the role, if any, of cyt-1 in apoptosis. It shares a promoter with ced-9 and may be involved in regulation of ced-9; also like bcl-2, it encodes for a mitochondrial protein.

\section{N S THAKKER}

\section{Medium-chain acyl-coenzyme A dehydrogenase deficiency: clinical course in 120 affected children \\ Kimberly A, Lafolla Y, Thompson RJ Jr, Roe CR. F Pediatr 1994;124:409-15.}

Medium-chain acyl-coenzyme A dehydrogenase (MCAD) deficiency is an autosomal recessive disorder of fatty acid oxidation with an incidence of 1 in 23000 . It is found almost exclusively in persons of northern European origin. In the majority of cases there is a common mutation, G985, in the MCAD gene on chromosome 1 , and so screening for this disorder by molecular analysis is relatively straightforward. In recent years, MCAD deficiency has been shown to be a cause of sudden infant death syndrome (SIDS), but the outcome is by no means always fatal and it may also present as episodic hypoglycaemia, encephalopathy, and apnoeic attacks in childhood. Treatment involves avoidance of hypoglycaemia and a low fat diet. In this regimen the symptoms are usually self limiting and the course benign. In this large study of $120 \mathrm{MCAD}$ deficiency patients some interesting facts come to light: eighty-eight percent of patients were initially referred because of clinical illness or sudden death. Viral infections precipitated the illness in $85 \%$ cases. Only $12 \%$ were initially suspected of having MCAD deficiency. Other initial diagnoses included Reye's syndrome, SIDS, idiopathic hypoglycaemia, and carnitine deficiency. Twenty percent of patients died, and in addition to fatty changes in the liver, heart, and kidneys, notable post mortem findings included small adrenal glands and a high frequency of stress ulceration of the gastrointestinal tract. Ninety-seven patients were followed up. Of these, $29 \%$ went on to have further episodes and developed other problems including muscle weakness, seizures, failure to thrive, and "cerebral palsy" and $40 \%$ had developmental problems which were often mild but included acquired aphasia, global developmental delay, and behavioural abnormalities. This group were more likely to have had seizures, encephalopathy, and hyperammonaemia and to have suffered from multiple episodes. The problems only appeared to develop after the first episode. Twenty-three living sibs were affected on screening and 14 had died. Five carrier parents had been symptomatic in childhood. Clearly, routine testing of sibs is indicated but the authors also suggest that MCAD deficiency satisfies the criteria for newborn population screening. The findings of this study lead us to consider whether we should be screening for MCAD deficiency and for carrier status in children with apparently acquired developmental problems, especially acquired aphasia and attention deficit disorder.

\section{JILL CLAYTON-SMITH}

Gonosomal mosaicism in myotonic dystrophy patients: involvement of mitotic events in (CTG), repeat variation and selection against extreme expansion in sperm

Jansen G, Willems $\mathrm{P}$, Coerwinkel $\mathrm{M}$, et al. Am $\mathcal{F}$ Hum Genet 1993;54:575-85.

Myotonic dystrophy (DM) is caused by the abnormal expansion of a polymorphic triplet $(C T G)_{n}$ repeat, which is located in the protein kinase gene. It is well known that the size of the triplet repeat in different offspring of an affected person can vary considerably, and intergenerational expansion is commone than intergenerational contraction. In this report, the length of the (CTG) ${ }_{n}$ repeat was determined in DNA from a wide range of tissues from patients with mild, classical, or congenital DM. The gene is expressed predominantly in smooth, skeletal, and hear muscle, and at low levels in brain and endocrine tissues. This pattern of expression parallels the tissues affected clinically. In norma persons the (CTG) repeat varies between three and 37 repeat units, with clusters at five, 11-14, and 18-22 CTGs. In affected persons repeats up to 100 units appeared as defined bands on Southern blot analysis, but larger repeat alleles (some over 1000 units) appeared as a diffuse smear because of mitotic instability. Differences in repeat length were seen in somatic tissues from single DM persons (for example, ranging from 1000 to 1300 CTGs) and twins. Repeats expanded to a similar extent in different tissues of the same embryonal origin. In most male patients with small or intermediate sized expansions in blood DNA, the repeat lengths covered a much wider range in sperm. In contrast, male patients with large allele expansions ( $>700$ CTGs) in blood had similar or smaller repeats in sperm. Sperm alleles with 1000 CTGs were not seen, suggesting selection against extreme expansion in sperm. The most remarkable contraction involved a paternal allele of approximately 400 CTGs, which underwent compression to 24 CTGs in the offspring (non-paternity was excluded). The authors conclude that DM patients seem to be "gonosomal mosaics", that is, combined somatic and germline mosaics. Multiple cases are reported where the triplet repeat length in the offspring was larger than in the sperm, suggesting that intergenerational length changes in the unstable (CTG) repeat are most likely to occur during early embryonic mitotic divisions in both somatic and germline tissue formation. The initial size of the (CTG) ${ }_{n}$ repeat, the overall number of cell divisions involved in tissue formation, and a specific selection process in spermatogenesis may all influence the variation in repeat size.

\section{FRANCES FLINTER}

Somatic mutations and cellular selection in paroxysmal nocturnal haemoglobinuria

Bessler M, Mason P, Hillmen, P, Luzzatto L. Lancet 1994;343:951-3.

Chronic intravascular haemolysis is the clincal hallmark of paroxysmal nocturnal haemoglobinuria (PNH) and is the result of ncreased complement sensitivity of a mutant cell line. The molecular basis of PNH has been shown to be alterations in the PIG-A gene on $\mathrm{Xp}$. Received wisdom suggests that the somatic mutation takes place early in haematopoietic stem cell development. Although the relationship between the mutant cell clone and the normal cell population is unclear, the considerable expansion in the mutant line which must precede clinical signs has been proposed as evidence for positive selection and survival advantage of the mutant cell line relative to normal haematopoiesis. Bessler et al show, in an elegan series of experiments, that two patients with PNH have two independently arising PNH clonal lines each. All four clones have an entirely separate mutational basis. They argue that these observations further support positive selection of PNH clones with inhibition of normal haematopoiesis. With regard to the known association between PNH and aplastic anaemia, their suggestion is that aplastic anaemia inhibits normal haematopoiesis but that PNH cell clones are unaffected by this inhibition. Haematopoiesis, albeit of an abnormal clone continues, an example of gene therapy in the wild. Medical school recollections of PNH are of a condition, relevant'only to the MRCP part 1 MCQ examination, which made little biological sense. These authors have clarified the apparent eccentricities of the condition. In doing so, they also show that elegance in the laboratory and elegance on the page are not synonymous. 